\title{
PARITY-REGULAR STEINHAUS GRAPHS
}

\author{
MAXIME AUGIER AND SHALOM ELIAHOU
}

\begin{abstract}
Steinhaus graphs on $n$ vertices are certain simple graphs in bijective correspondence with binary $\{0,1\}$-sequences of length $n-1$. A conjecture of Dymacek in 1979 states that the only nontrivial regular Steinhaus graphs are those corresponding to the periodic binary sequences $110 \ldots 110$ of any length $n-1=3 m$. By an exhaustive search the conjecture was known to hold up to 25 vertices. We report here that it remains true up to 117 vertices. This is achieved by considering the weaker notion of parity-regular Steinhaus graphs, where all vertex degrees have the same parity. We show that these graphs can be parametrized by an $\mathbb{F}_{2}$-vector space of dimension approximately $n / 3$ and thus constitute an efficiently describable domain where true regular Steinhaus graphs can be searched by computer.
\end{abstract}

\section{INTRODUCTION}

Let $s=a_{1} \ldots a_{n-1}$ be a binary sequence of length $n-1$ with entries $a_{i}$ in the 2-element field $\mathbb{F}_{2}=\{0,1\}$. The Steinhaus graph associated with $s$ is the simple graph $G(s)$ on the vertex set $\{0,1, \ldots, n-1\}$ whose adjacency matrix $M(s)=$ $\left(a_{i, j}\right) \in \mathcal{M}_{n}\left(\mathbb{F}_{2}\right)$, with indices $0 \leq i, j \leq n-1$, is defined as follows:

1. $a_{i, i}=0$ for $0 \leq i \leq n-1$,

2. $a_{0, i}=a_{i}$ for $1 \leq i \leq n-1$,

3. $a_{i, j}=a_{i-1, j-1}+a_{i-1, j}$ for $1 \leq i<j \leq n-1$,

4. $a_{j, i}=a_{i, j}$ for $0 \leq i<j \leq n-1$.

Note that the first row of $M(s)$ is the vector $\left(0, a_{1}, \ldots, a_{n-1}\right)$, and that each subsequent row is determined, in its strict upper triangular part, by its predecessor using rule 3 . For example, if $s=a_{1} \ldots a_{4}$, then

$$
M(s)=\left(\begin{array}{ccccc}
0 & a_{1} & a_{2} & a_{3} & a_{4} \\
a_{1} & 0 & a_{1}+a_{2} & a_{2}+a_{3} & a_{3}+a_{4} \\
a_{2} & a_{1}+a_{2} & 0 & a_{1}+a_{3} & a_{2}+a_{4} \\
a_{3} & a_{2}+a_{3} & a_{1}+a_{3} & 0 & a_{1}+a_{2}+a_{3}+a_{4} \\
a_{4} & a_{3}+a_{4} & a_{2}+a_{4} & a_{1}+a_{2}+a_{3}+a_{4} & 0
\end{array}\right) .
$$

The strict upper triangular part of $M(s)$ is known as the Steinhaus triangle associated with $s$, first defined by Steinhaus in [7. We say that the graph $G(s)$ is generated by the binary sequence $s$. Steinhaus graphs (in fact, their complements) were introduced by John Molluzzo in [6]. It can easily be shown [3] that all Steinhaus graphs are connected, except those generated by the constant sequences $0 . .0$.

Received by the editor February 2, 2006 and, in revised form, April 13, 2007. 2000 Mathematics Subject Classification. Primary 11B75, 05C07, 05C50.

(C)2007 American Mathematical Society Reverts to public domain 28 years from publication 
Here are two easy examples of Steinhaus graphs: if $s=1$ of length 1 , then $G(s)=K_{2}$, the one-edge graph on 2 vertices; if $s=0 \ldots 0$ of length $n-1$ (also denoted $s=0^{n-1}$ ), then $G(s)$ is the zero-edge graph on $n$ vertices. These particular instances are regular graphs.

A general problem on Steinhaus graphs is that of characterizing those satisfying a given graph property. For instance, all bipartite and all planar Steinhaus graphs are now known [2, 4]. As for regular Steinhaus graphs, it is believed that the currently known ones exhaust them all. Besides the trivial instances mentioned above, namely $G(1)=K_{2}$ and $G\left(0^{n-1}\right)$, there is also a nontrivial infinite family of regular Steinhaus graphs on $n=3 m+1$ vertices, generated by the periodic sequence $s=110 \ldots 110$ of length $3 m(m \geq 1)$. It is an amusing exercise to check that $G\left((110)^{m}\right)$ is indeed regular of degree $2 m$.

Dymacek first conjectured in 1979 that there are no other regular Steinhaus graphs besides those described above [3. Bailey and Dymacek verified this conjecture for $n \leq 25$ vertices in 1988 (see [1]). In this paper we extend the verification as follows.

Computational Result 1. There are no other regular Steinhaus graphs on $n \leq$ 117 vertices besides $G(1), G\left(0^{n-1}\right)$ and $G\left((110)^{m}\right)$.

This is achieved by computer-searching regular Steinhaus graphs inside the larger class of parity-regular Steinhaus graphs, which, for $n$ vertices, are proved to depend on at most $\lceil n / 3\rceil$ binary parameters.

\section{PARITY-REgular GRAPHS}

We shall say that a locally finite graph is parity-regular if its vertex degrees all have the same parity. For instance, regular graphs are parity-regular. We say that a parity-regular graph $G$ is of odd type if its vertex degrees are all odd, and of even type otherwise. Note that finite parity-regular graphs of even type are also known as even graphs, or Eulerian graphs if connected.

It is well known that in a finite graph, the number of vertices of odd degree is even. This implies the following.

Lemma 1. There are no parity-regular graphs of odd type with an odd number of vertices.

Proof. If $V$ and $E$ denote the vertex set and the edge set, respectively, then $2|E|=$ $\sum_{x \in V} \operatorname{deg}(x) \equiv|V| \bmod 2$.

We now show that parity-regular Steinhaus graphs on $n$ vertices can be parametrized by approximately $n / 3$ binary parameters. This fact greatly accelerates the search for true regular Steinhaus graphs.

Theorem 2. Let $n \geq 2$ be an integer. The set of parity-regular Steinhaus graphs on $n$ vertices is in bijection with a vector space over $\mathbb{F}_{2}$ of dimension $\left\lceil\frac{n}{3}\right\rceil-\varepsilon(n)$, where $\varepsilon(k)=0$ or 1 and $\varepsilon(k) \equiv k \bmod 2$.

Proof. Let $s=a_{1} \ldots a_{n-1}$ be a binary sequence of length $n-1$, or equivalently let $s \in \mathbb{F}_{2}^{n-1}$. Let $L_{i}(s) \in \mathbb{F}_{2}$ denote the $i$ th row coefficient sum of the associated 
matrix $M(s)$, for each $i=0, \ldots, n-1$. Thus, we have

$$
M(s) \cdot\left(\begin{array}{c}
1 \\
\vdots \\
1
\end{array}\right)=\left(\begin{array}{c}
L_{0}(s) \\
\vdots \\
L_{n-1}(s)
\end{array}\right)
$$

as matrices with coefficients in $\mathbb{F}_{2}$. For example, if $n=5$, then $L_{0}(s)=a_{1}+a_{2}+$ $a_{3}+a_{4}, L_{1}(s)=a_{4}, L_{2}(s)=a_{2}+a_{3}+a_{4}, L_{3}(s)=a_{4}$ and $L_{4}(s)=a_{1}$. (Compare with the matrix shown in the Introduction.)

Let $R(n)=\left\{s \in \mathbb{F}_{2}^{n-1}: L_{i}(s)=L_{j}(s)\right.$ for $\left.0 \leq i, j \leq n-1\right\}$. Then $R(n)$ is a subspace of $\mathbb{F}_{2}^{n-1}$, as the $L_{i}(s)$ are linear forms in $s$. It easily follows from the definitions that the graph $G(s)$ is parity-regular if and only if $s \in R(n)$. Thus, the subspace $R(n)$ corresponds bijectively to the set of parity-regular Steinhaus graphs on $n$ vertices.

It is convenient to decompose $R(n)$ as the disjoint union $R_{0}(n) \cup R_{1}(n)$, where $R_{\lambda}(n)=\left\{s \in \mathbb{F}_{2}^{n-1}: L_{i}(s)=\lambda\right.$ for $\left.0 \leq i \leq n-1\right\}$ for $\lambda \in \mathbb{F}_{2}=\{0,1\}$.

The set $R_{0}(n)$ is a subspace of $R(n)$, corresponding to even 1 Steinhaus graphs on $n$ vertices. The set $R_{1}(n)$ corresponds to the odd type, and is either empty or an affine translate of $R_{0}(n)$. It follows from Lemma 1 that $R_{1}(n)=\emptyset$ if $n$ is odd.

The dimension count of $R(n)$ is made possible by wonderful results in [3]. There, Dymacek shows (Theorem 3.5, page 409) that the set of even Steinhaus graphs on $n$ vertices is of cardinality $2^{\left\lfloor\frac{n-1}{3}\right\rfloor}$. This is equivalent to the formula $\operatorname{dim} R_{0}(n)=$ $\left\lfloor\frac{n-1}{3}\right\rfloor$. He further shows (Corollary 3.7, page 411) that for $n$ even, there are also $2^{\left\lfloor\frac{n-1}{3}\right\rfloor}$ Steinhaus graphs on $n$ vertices with all vertices of odd degree (i.e., parityregular of odd type, in our terminology). Thus, for $n$ even, the set $R_{1}(n)$ is an affine translate of the subspace $R_{0}(n)$. For $n$ odd, we have already observed that $R_{1}(n)$ is empty. Hence, for the disjoint union $R(n)=R_{0}(n) \cup R_{1}(n)$, we get

$$
|R(n)|= \begin{cases}2^{\left\lfloor\frac{n-1}{3}\right\rfloor} & \text { if } n \text { is odd } \\ 2^{\left\lfloor\frac{n-1}{3}\right\rfloor+1} & \text { if } n \text { is even. }\end{cases}
$$

This yields the formula $\operatorname{dim} R(n)=\left\lfloor\frac{n-1}{3}\right\rfloor+\varepsilon(n-1)$. It is a simple matter to see that $\left\lfloor\frac{n-1}{3}\right\rfloor+\varepsilon(n-1)=\left\lceil\frac{n}{3}\right\rceil-\varepsilon(n)$ for all $n \geq 1$, as witnessed by the following table:

\begin{tabular}{|c||c|c|c|c|c|c|}
\hline$n$ & $6 k$ & $6 k+1$ & $6 k+2$ & $6 k+3$ & $6 k+4$ & $6 k+5$ \\
\hline \hline$\left\lfloor\frac{n-1}{3}\right\rfloor+\varepsilon(n-1)$ & $2 k$ & $2 k$ & $2 k+1$ & $2 k$ & $2 k+2$ & $2 k+1$ \\
\hline$\left\lceil\frac{n}{3}\right\rceil-\varepsilon(n)$ & $2 k$ & $2 k$ & $2 k+1$ & $2 k$ & $2 k+2$ & $2 k+1$ \\
\hline
\end{tabular}

As a last remark, note that $\operatorname{dim} R(n)$ satisfies the relation

$$
\operatorname{dim} R(n+6)=\operatorname{dim} R(n)+2
$$

for all $n \geq 1$.

\footnotetext{
${ }^{1}$ As observed earlier, all Steinhaus graphs are connected except the zero-edge ones. Thus, nontrivial even Steinhaus graphs are actually Eulerian.
} 


\section{Parametrizations uP to 30 vertices}

As an illustration of Theorem 2, here we give the parametrizations of all parityregular Steinhaus graphs up to 30 vertices. For a given number $n$ of vertices, the sequence $s=\left(x_{1}, \ldots, x_{n-1}\right) \in \mathbb{F}_{2}^{n-1}$ yields a parity-regular Steinhaus graph if and only if $s$ is of the form shown below. Observe that the number of free parameters is everywhere $\left\lceil\frac{n}{3}\right\rceil-\varepsilon(n)$, as expected. The cases $n=7$ and $n=9$ are illustrated in the next section.

These parametrizations were obtained by a simple Mathematica program, in the spirit of the one given in [5]. One key function is GroebnerBasis, used to perform Gaussian elimination on the family of linear forms $L_{i}(s)-L_{j}(s)$ defining the set $R(n)$ in the proof of Theorem 2, Our program easily produces such parametrizations for $n$ in the hundreds.

$$
\begin{aligned}
& n=2:\left(x_{1}\right) \\
& n=3:(0,0) \\
& n=4:\left(x_{1}, x_{1}, x_{3}\right) \\
& n=5:\left(0, x_{2}, x_{2}, 0\right) \\
& n=6:\left(x_{1}, x_{1}, x_{1}, x_{1}, x_{5}\right) \\
& n=7:\left(x_{1}, x_{2}, x_{1}+x_{2}, x_{2}, x_{2}, 0\right) \\
& n=8:\left(x_{1}, x_{2}, x_{2}, x_{1}, x_{2}, x_{2}, x_{7}\right) \\
& n=9:\left(0, x_{2}, x_{3}, x_{2}, x_{3}, x_{2}, x_{2}, 0\right) \\
& n=10:\left(x_{1}, x_{1}, x_{3}, x_{4}, x_{1}, x_{1}+x_{3}+x_{4}, x_{1}, x_{1}, x_{9}\right) \\
& n=11:\left(x_{1}, x_{2}, x_{1}+x_{2}, x_{4}, x_{1}+x_{2}, x_{2}, x_{1}+x_{4}, x_{2}, x_{2}, 0\right) \\
& n=12:\left(x_{1}, x_{2}, x_{2}, x_{1}, x_{5}, x_{1}+x_{2}+x_{5}, x_{2}, x_{1}, x_{2}, x_{2}, x_{11}\right) \\
& n=13:\left(x_{1}, x_{2}, x_{3}, x_{2}, x_{1}+x_{3}, x_{6}, x_{2}+x_{3}+x_{6}, x_{2}, x_{3}, x_{2}, x_{2}, 0\right) \\
& n=14:\left(x_{1}, x_{2}, x_{3}, x_{4}, x_{2}, x_{1}+x_{3}+x_{4}, x_{1}+x_{2}+x_{3}, x_{1}+x_{2}+x_{4}, x_{2}, x_{2}\right. \\
& \left.+x_{3}+x_{4}, x_{2}, x_{2}, x_{13}\right) \\
& n=15:\left(x_{1}, x_{2}, x_{3}, x_{4}, x_{3}, x_{2}, x_{1}+x_{4}, x_{4}, x_{3}, x_{2}, x_{2}+x_{3}+x_{4}, x_{2}, x_{2}, 0\right) \\
& n=16:\left(x_{1}, x_{2}, x_{3}, x_{4}, x_{5}, x_{3}+x_{4}+x_{5}, x_{2}, x_{1}, x_{2}+x_{3}+x_{5}, x_{2}+x_{4}+x_{5}, x_{2}, x_{2}\right. \\
& \left.+x_{3}+x_{4}, x_{2}, x_{2}, x_{15}\right) \\
& n=17:\left(0, x_{2}, x_{3}, x_{4}, x_{5}, x_{6}, x_{3}+x_{4}+x_{6}, x_{2}, x_{5}, x_{2}+x_{4}+x_{6}, x_{2}+x_{3}+x_{6}, x_{2}, x_{2}\right. \\
& \left.+x_{3}+x_{4}, x_{2}, x_{2}, 0\right) \\
& n=18:\left(x_{1}, x_{1}, x_{3}, x_{4}, x_{5}, x_{6}, x_{4}+x_{5}+x_{6}, x_{3}+x_{5}+x_{6}, x_{1}, x_{1}+x_{5}+x_{6}, x_{1}\right. \\
& \left.+x_{4}+x_{6}, x_{1}+x_{3}+x_{6}, x_{1}, x_{1}+x_{3}+x_{4}, x_{1}, x_{1}, x_{17}\right) \\
& n=19:\left(x_{1}, x_{2}, x_{1}+x_{2}, x_{4}, x_{5}, x_{6}, x_{7}, x_{4}, x_{1}+x_{2}, x_{2}, x_{1}+x_{5}+x_{6}+x_{7}, x_{2}\right. \\
& \left.+x_{4}+x_{6}, x_{1}+x_{6}, x_{2}, x_{1}+x_{4}, x_{2}, x_{2}, 0\right) \\
& n=20:\left(x_{1}, x_{2}, x_{2}, x_{1}, x_{5}, x_{6}, x_{7}, x_{8}, x_{1}+x_{6}+x_{7}, x_{2}+x_{6}+x_{7}, x_{2}, x_{1}+x_{5}\right. \\
& \left.+x_{6}+x_{7}+x_{8}, x_{1}+x_{2}+x_{6}, x_{6}, x_{2}, x_{1}, x_{2}, x_{2}, x_{19}\right) \\
& n=21:\left(x_{1}, x_{2}, x_{3}, x_{2}, x_{1}+x_{3}, x_{6}, x_{7}, x_{8}, x_{1}+x_{3}, x_{2} \quad+\quad x_{6}\right. \\
& \left.+x_{8}, x_{3}+x_{6}+x_{8}, x_{2}, x_{1}+x_{6}+x_{7}+x_{8}, x_{6}, x_{2}+x_{3}+x_{6}, x_{2}, x_{3}, x_{2}, x_{2}, 0\right) \\
& n=22:\left(x_{1}, x_{2}, x_{3}, x_{4}, x_{2}, x_{1}+x_{3}+x_{4}, x_{7}, x_{8}, x_{9}, x_{1}+x_{2}+x_{3}+x_{4}+x_{9}, x_{1}\right. \\
& +x_{3}+x_{9}, x_{1}+x_{4}+x_{9}, x_{2}, x_{1}+x_{7}+x_{8}, x_{1}+x_{2}+x_{3}, x_{1}+x_{2}+x_{4}, x_{2}, x_{2}+ \\
& \left.x_{3}+x_{4}, x_{2}, x_{2}, x_{21}\right) \\
& n=23:\left(x_{1}, x_{2}, x_{3}, x_{4}, x_{3}, x_{2}, x_{1}+x_{4}, x_{8}, x_{9}, x_{10}, x_{1}+x_{2}+x_{3}+x_{4}+x_{9}\right. \\
& +x_{10}, x_{2}+x_{4}+x_{10}, x_{2}+x_{3}+x_{10}, x_{2}, x_{1}+x_{8}, x_{4}, x_{3}, x_{2}, x_{2}+x_{3}+x_{4}, x_{2} \text {, } \\
& \left.x_{2}, 0\right) \\
& n=24:\left(x_{1}, x_{2}, x_{3}, x_{4}, x_{5}, x_{3}+x_{4}+x_{5}, x_{2}, x_{1}, x_{9}, x_{10}, x_{2}+x_{3}+x_{4}+x_{5}\right. \\
& +x_{10}, x_{1}+x_{5}+x_{9}, x_{2}+x_{4}+x_{10}, x_{2}+x_{3}+x_{10}, x_{2}, x_{1}, x_{2}+x_{3}+x_{5}, x_{2}+x_{4} \\
& \left.+x_{5}, x_{2}, x_{2}+x_{3}+x_{4}, x_{2}, x_{2}, x_{23}\right)
\end{aligned}
$$




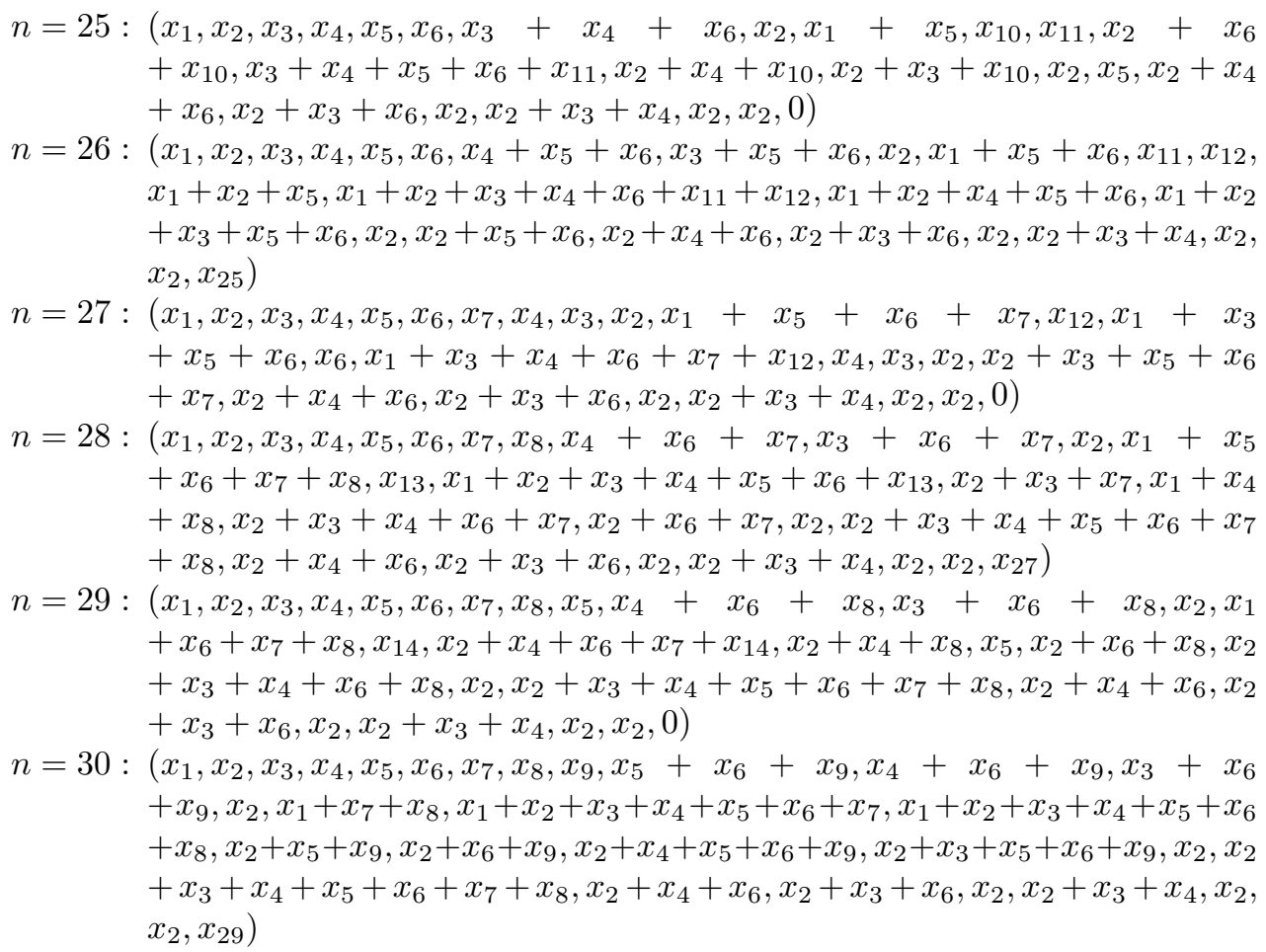

\section{THE CASES $n=7$ AND $n=9$}

We illustrate the above parametrizations with two examples, the cases $n=7$ and $n=9$. According to the preceding section, the complete list of parity-regular Steinhaus graphs $G(s)$ on 7 vertices is given by all sequences $s \in \mathbb{F}_{2}^{6}$ of the form $s=\left(x_{1}, x_{2}, x_{1}+x_{2}, x_{2}, x_{2}, 0\right)$ with $x_{1}, x_{2} \in \mathbb{F}_{2}$. Thus, there are exactly four such graphs. Among them, two are truly regular, namely for $x_{1}=x_{2}=0$, yielding $s=(0,0,0,0,0,0)$, and for $x_{1}=x_{2}=1$, yielding $s=(1,1,0,1,1,0)$.

For $n=9$, the complete list of parity-regular Steinhaus graphs on 9 vertices is given by all sequences $s \in \mathbb{F}_{2}^{8}$ of the form $s=\left(0, x_{2}, x_{3}, x_{2}, x_{3}, x_{2}, x_{2}, 0\right)$ with $x_{2}, x_{3} \in \mathbb{F}_{2}$. Again, there are four such graphs. But here, only the zero-edge one is truly regular. The other three graphs are pictured in Figure 1. (There, the vertices are denoted $1,2, \ldots, 9$ rather than $0,1, \ldots, 8)$.

\section{The MAIN RESUlT}

Using Theorem 2 and our explicit parametrizations of parity-regular Steinhaus graphs, we have been able to greatly extend the verification of the conjecture of Dymacek, by searching regular Steinhaus graphs inside the larger class of parityregular ones. Our result is as follows:

There are no new regular Steinhaus graphs on $n \leq 117$ vertices other than those already known, i.e. $G(1), G\left(0^{n-1}\right)$ and $G\left((110)^{\lfloor n / 3\rfloor}\right)$.

Recall from Section 2 that for any given $n$, the search space $R(n)$ is of size $2^{\lceil n / 3\rceil-\varepsilon(n)}$. Up to $n \leq 81$ vertices, our search was performed in a few days on a standard PC running a simple uncompiled Mathematica 5.0 program. 

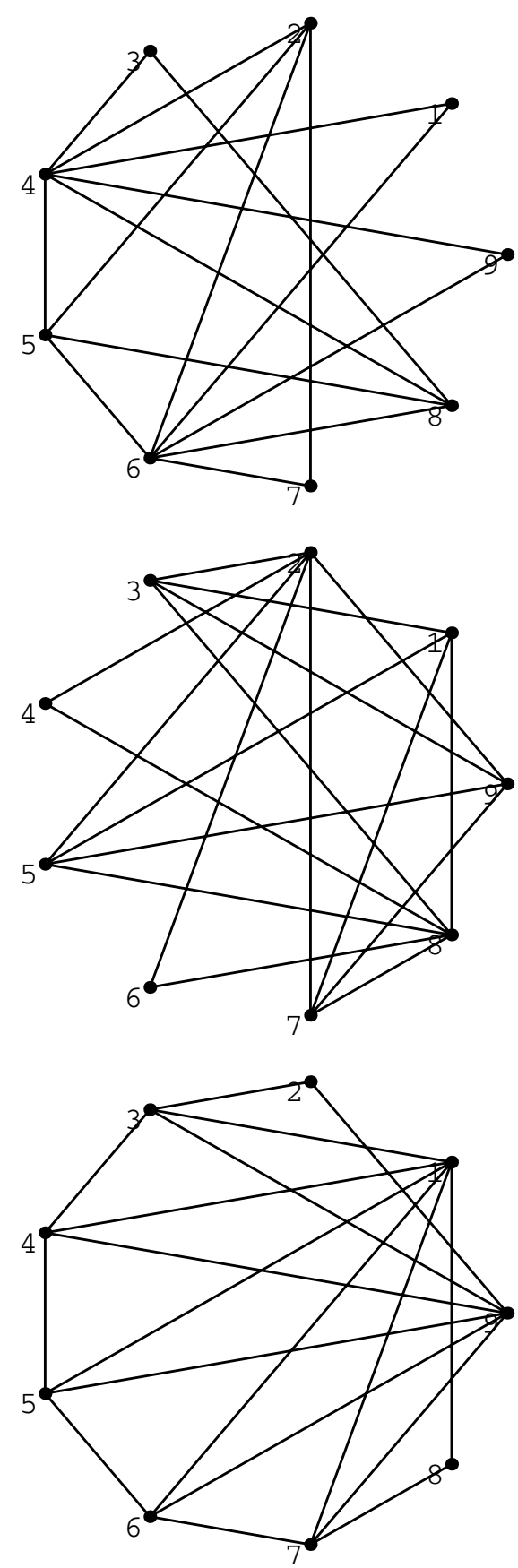

FiguRE 1. All nontrivial parity-regular Steinhaus graphs on 9 vertices 
However, to treat the range $82 \leq n \leq 117$, we had to switch to a carefully written and highly optimized program in $\mathrm{C}$. This computation was run on a 16-processor Bull NovaScale machine in less than two weeks.

\section{Related PRoblems}

Two interesting problems arose during our investigations on parity-regular Steinhaus graphs. As for regularity, they both concern the vertex degree distribution of such graphs. For convenience, we shall sometimes write "PRS graph" for "parityregular Steinhaus graph" in this section.

Question 1. Are there parity-regular Steinhaus graphs on $n$ vertices with exactly two distinct vertex degrees?

For even $n$, the following binary sequences yield solutions to Question 1:

- If $s=0^{n-2} 1$ or $1^{n-1}$, the set of vertex degrees of $G(s)$ is $\{1, n-1\}$.

- If $s=1^{n-2} 0$, the set of vertex degrees of $G(s)$ is $\{2, n-2\}$.

The hypothesis that $n$ is even ensures that the above graphs are indeed parityregular. There are other systematic solutions, depending on the class of $n \bmod$ 6 :

- If $n \equiv 1 \bmod 6$ and $s=(101000)^{(n-1) / 6}$, then the degree set of $G(s)$ is $\{k, 2 k\}$ where $k=\frac{n-1}{3}$.

- If $n \equiv 2$ or $5 \bmod 6$ and $s=0(110)^{(n-2) / 3}$, then $G(s)$ has degree set equal to $\{2 k, 2 k+2\}$ where $k=\frac{n-2}{3}$.

Surprisingly, in the remaining case $n \equiv 3 \bmod 6$, there seem to be $n o$ solutions, except for $n=3$ and $n=15$. We have verified this up to $n \leq 45$ vertices.

Conjecture 1. For every $n \equiv 3 \bmod 6$ with $n \geq 21$, there are no parity-regular Steinhaus graphs on $n$ vertices having exactly two distinct vertex degrees.

In the same type of question, we can show that, for every $n \geq 9$, there are $P R S$ graphs on $n$ vertices with exactly three distinct degrees. Solutions $s \in \mathbb{F}_{2}^{n-1}$ of the form $0(1)^{2 k} 0,(001)^{2 k+1}, 1(001)^{2 k},(0110)^{2 k} 110$ and $0(0110)^{2 k+1} 0^{4}$ suffice to cover all $n \geq 9$.

Our second problem concerns the reconstructibility of PRS graphs from their degree distributions. We denote by $d_{i}$ the degree of vertex $i$.

Question 2. Is it true that a parity-regular Steinhaus graph on $n$ vertices is determined by its degree sequence $\left(d_{0}, d_{1}, \ldots, d_{n-1}\right)$ ?

For example, there are exactly four PRS graphs on $n=6$ vertices. Their respective degree sequences are $(0,0,0,0,0,0),(1,1,1,1,1,5),(5,1,1,1,1,1)$ and $(4,2,2,2,2,4)$. These four sequences being distinct, Question 2 has a positive answer for $n=6$.

While the answer to Question 2 turns out to be negative in general, counterexamples seem to be very rare. To facilitate their discussion, we define a collision to be a pair of distinct binary sequences $s_{1}, s_{2} \in \mathbb{F}_{2}^{n-1}$ such that

- the associated Steinhaus graphs $G\left(s_{1}\right), G\left(s_{2}\right)$ are parity-regular, and

- the degree sequences of $G\left(s_{1}\right), G\left(s_{2}\right)$ are identical. 
The smallest collision occurs at $n=26$ vertices. It is given by the pair $s_{1}, s_{2} \in$ $\mathbb{F}_{2}^{25}$, where

$$
\begin{aligned}
& s_{1}=(0,0,1,0,1,0,1,0,0,1,1,1,1,1,1,0,0,1,0,1,0,1,0,0,1) \\
& s_{2}=(1,1,0,1,0,1,0,1,1,0,0,0,0,0,0,1,1,0,1,0,1,0,1,1,1) .
\end{aligned}
$$

Observe the relation $s_{1}+s_{2}=(1,1, \ldots, 1,0) \in \mathbb{F}_{2}^{25}$. The common degree sequence of their associated Steinhaus graphs $G\left(s_{1}\right), G\left(s_{2}\right)$ is

$(13,15,9,9,13,13,17,11,9,19,9,9,11,11,9,9,19,9,11,17,13,13,9,9,15,13)$.

(As required, all vertex degrees have the same parity.)

This is the sole collision for $n \leq 26$. We found that, up to 50 vertices, the only other collisions occur at $n=34,38,42,46$ and 50. Moreover, each one satisfies the relation $s_{1}+s_{2}=(1,1, \ldots, 1,0) \in \mathbb{F}_{2}^{n-1}$. Below we give the complete list of collisions $s_{1}, s_{2}$ up to $n \leq 50$ vertices. As these pairs all satisfy $s_{2}=s_{1}+(1,1, \ldots, 1,0)$, we display $s_{1}$ only, in the form of a binary string.

$$
\begin{aligned}
& n=26: \quad 0010101001111110010101001 \\
& n=34: \quad 000011101110110000110111011100001 \\
& n=38: \quad 0000001011110110111101101111010000001 \\
& n=42: \quad 00001100001111101110011101111100001100001 \\
& 00100110010011101011110101110010011001001 \\
& 00101010011100100111111001001110010101001 \\
& 00101010011111000101101000111110010101001 \\
& 00101010011111001010010100111110010101001 \\
& 00101010011111101000000101111110010101001 \\
& n=46: \quad 000000101111010011110110111100101111010000001 \\
& 000011100011010011101111011100101100011100001 \\
& 001001100110010010111111110100100110011001001 \\
& 001010101010010001111111111000100101010101001 \\
& n=50: \quad 000011001100110000111111111110000110011001100001 \\
& 0000111011101100001101011010110000110111011100001 \\
& 0000111011101100001110011001110000110111011100001 \\
& 0010011010010110010011111111001001101001011001001 \\
& 0010011010110010011001111110011001001101011001001 \\
& 0010011010110010011010111101011001001101011001001 \\
& 0010100001011110010111100111101001111010000101001 \\
& 0010100001111010011101100110111001011110000101001 \\
& 0010100001111010011110100101111001011110000101001 \\
& 0010100010101110010111011011101001110101000101001 \\
& 0010101001011010011111000011111001011010010101001 \\
& 0010101001111110010101000010101001111110010101001 \\
& 0010101001111110010110000001101001111110010101001 \\
& 0010101010001110010101111110101001110001010101001 \\
& 0010101010001110010110111101101001110001010101001 \\
& 0010101010101010011100111100111001010101010101001
\end{aligned}
$$

This leads us to the following conjecture.

Conjecture 2. If $s_{1}, s_{2} \in \mathbb{F}_{2}^{n-1}$ is a collision, then $n \equiv 2 \bmod 4$ and $s_{1}+s_{2}=$ $(1,1, \ldots, 1,0)$. 
In other words, we conjecture that whenever $n \not \equiv 2 \bmod 4$, a parity-regular Steinhaus graph on $n$ vertices is completely determined by its vertex degree sequence. If true, it would be extremely interesting to know how to reconstruct the sequence $s$ from the degree sequence of $G(s)$.

\section{ACKNOWLEDGMENTS}

We thank Jarik Nešetřil for suggesting us the term parity-regular. The second author gratefully acknowledges partial support from the Fonds National Suisse de la Recherche Scientifique during the preparation of this paper.

\section{REFERENCES}

[1] C. Bailey and W. Dymacek, Regular Steinhaus graphs, Proc. 19th southeast. Conf. Combinatorics, Graph Theory and Computing, Baton Rouge 1988, Congr. Numerantium 66 (1988) 45-47. MR992886 (90a:05132)

[2] G.J. Chang, B. DasGupta, W. Dymacek, M. Fürer, M. Koerlin, Y-S. Lee and T. Whaley, Characterizations of bipartite Steinhaus graphs, Discrete Math. 199, Nos. 1-3 (1999) 11-25. MR1675907 (2000a:05168)

[3] W. Dymacek, Steinhaus graphs, Proc. 10th southeast. Conf. Combinatorics, Graph Theory and Computing, Boca Raton 1979, Vol. I, Congr. Numerantium 23 (1979) 399-412. MR561065 (81f:05120)

[4] W. Dymacek, J-G. Speton and T. Whaley, Planar Steinhaus graphs, Congr. Numerantium 144 (2000) 193-206. MR1817934 (2001m:05222)

[5] S. Eliahou and D. Hachez, On a problem of Steinhaus concerning binary sequences, Experimental Mathematics 13, No. 2 (2004) 215-229. MR2068895

[6] J. Molluzzo, Steinhaus graphs, Theor. Appl. Graphs, Proc. Kalamazoo 1976, Lect. Notes Math. 642 (1978) 394-402. MR499509 (80e:05092)

[7] H. Steinhaus, One Hundred Problems in Elementary Mathematics. Elinsford, NY: Pergamon, 1963. New York: Dover, 1979 (reprint). MR0157881(28:1110)

Ecole Polytechnique Federale de Lausanne, Lausanne, Switzerland

E-mail address: maxime.augier@epfl.ch

LMPA-UlCO, B.P. 699, 62228 Calais, Cedex France

E-mail address: eliahou@lmpa.univ-littoral.fr 